\title{
Long-Term Monitoring of Bright Blazars in the Multi-GeV to TeV Range with FACT
}

\author{
Fabian Temme 1,*, Jan Adam ${ }^{1}$, Max L. Ahnen ${ }^{2}$, Dominik Baack ${ }^{1}$, Matteo Balbo ${ }^{3}$, \\ Matthias Bergmann ${ }^{4}$, Adrian Biland ${ }^{2}$, Michael Blank ${ }^{4}$, Thomas Bretz ${ }^{2}$, Kai A. Brügge ${ }^{1}$, \\ Jens Buss ${ }^{1}$, Anton Dmytriiev ${ }^{3}$, Daniela Dorner ${ }^{4}$, Sabrina Einecke ${ }^{1}$, Christina Hempfling ${ }^{4}$, \\ Dorothee Hildebrand ${ }^{2}$, Gareth Hughes ${ }^{2}$, Lena Linhoff ${ }^{1}$, Karl Mannheim ${ }^{4}$, Sebastian Müller ${ }^{2}$, \\ Dominik Neise ${ }^{2}$, Andrii Neronov ${ }^{3}$, Max Nöthe ${ }^{1}$, Aleksander Paravac ${ }^{4}$, Felicitas Pauss ${ }^{2}$, \\ Wolfgang Rhode ${ }^{1}$, Amit Shukla ${ }^{2}$, Julia Thaele ${ }^{1}$ and Roland Walter ${ }^{3}$
}

1 TU Dortmund, Experimental Physics 5, Otto-Hahn-Str. 4, 44221 Dortmund, Germany; jan.adam@udo.edu (J.A.); dominik.baack@tu-dortmund.de (D.B.); kai.bruegge@tu-dortmund.de (K.A.B.); jens.buss@tu-dortmund.de (J.B.); sabrina.einecke@uni-dortmund.de (S.E.);

lena.linhoff@tu-dortmund.de (L.L.); max.noethe@t-online.de (M.N.); rhode@physik.tu-dortmund.de (W.R.); julia.thaele@tu-dortmund.de (J.T.)

2 ETH Zurich, Institute for Particle Physics, Otto-Stern-Weg 5, 8093 Zurich, Switzerland; m.knoetig@gmail.com (M.L.A.); biland@phys.ethz.ch (A.B.); tbretz@physik.rwth-aachen.de (T.B.); dorothee.hildebrand@phys.ethz.ch (D.H.); ghughes@phys.ethz.ch (G.H.); sebmuell@phys.ethz.ch (S.M.); neised@phys.ethz.ch (D.N.); felicitas.pauss@cern.ch (F.P.); amit008@gmail.com (A.S.)

3 ISDC Data Center for Astrophysics, University of Geneva, Chemin d'Ecogia 16, 1290 Versoix, Switzerland; matteo.balbo@unige.ch (M.B.); anton.dmytriiev@etu.unige.ch (A.D.); Andrii.Neronov@unige.ch (A.N.); Roland.Walter@unige.ch (R.W.)

4 Institute for Theoretical Physics and Astrophysics, Universität Würzburg, Emil-Fischer-Str. 31, 97074 Würzburg, Germany; matthias.bergmann@stud-mail.uni-wuerzburg.de (M.B.); michael.blank@stud-mail.uni-wuerzburg.de (M.B.); dorner@astro.uni-wuerzburg.de (D.D.); christina.hempfling@uni-wuerzburg.de (C.H.); asti070@uni-wuerzburg.de (K.M.); aparavac@astro.uni-wuerzburg.de (A.P.)

* Correspondence: fabian.temme@tu-dortmund.de

Academic Editors: Jose L. Gómez, Alan P. Marscher and Svetlana G. Jorstad Received: 14 July 2016; Accepted: 2 February 2017; Published: 20 February 2017

\begin{abstract}
Blazars like Markarian 421 or Markarian 501 are active galactic nuclei (AGN), with their jets orientated towards the observer. They are among the brightest objects in the very high energy (VHE) gamma ray regime ( $>100 \mathrm{GeV})$. Their emitted gamma-ray fluxes are extremely variable, with changing activity levels on timescales between minutes, months, and even years. Several questions are part of the current research, such as the question of the emission regions or the engine of the AGN and the particle acceleration. A dedicated longterm monitoring program is necessary to investigate the properties of blazars in detail. A densely sampled and unbiased light curve allows for observation of both high and low states of the sources, and the combination with multi-wavelength observation could contribute to the answer of several questions mentioned above. FACT (First G-APD Cherenkov Telescope) is the first operational telescope using silicon photomultiplier (SiPM, also known as Geigermode-Avalanche Photo Diode, G-APD) as photon detectors. SiPM have a very homogenous and stable longterm performance, and allow operation even during full moon without any filter, leading to a maximal duty cycle for an Imaging Air Cherenkov Telescope (IACT). Hence, FACT is an ideal device for such a longterm monitoring of bright blazars. A small set of sources (e.g., Markarian 421, Markarian 501, 1ES 1959+650, and 1ES 2344+51.4) is currently being monitored. In this contribution, the FACT telescope and the concept of longterm monitoring of bright blazars will be introduced. The results of the monitoring program will be shown, and the advantages of densely sampled and unbiased light curves will be discussed.
\end{abstract}


Keywords: Imaging Air Cherenkov Telescope; First G-APD Cherenkov Telescope; very high energy gamma rays; long-term monitoring; silicon photo multiplier

\section{Introduction to the First G-APD Cherenkov Telescope}

FACT (Figure 1) is the first IACT which uses SiPM (also known as G-APD) instead of the commonly used PMT as photo sensors. The telescope is located at the Observatorio Roque de los Muchachos on the Canary island of La Palma, Spain, at about $2200 \mathrm{~m}$ above sea level. The novel camera was installed in October 2011 on the refurbished mount of the former HEGRA CT3 telescope and has successfully taken data since then. The mirror surface sums up to $9.5 \mathrm{~m}^{2}$ and the field of view of the camera is $4.5^{\circ}$. The camera is equipped with 1440 pixels, each consisting of a SiPM.

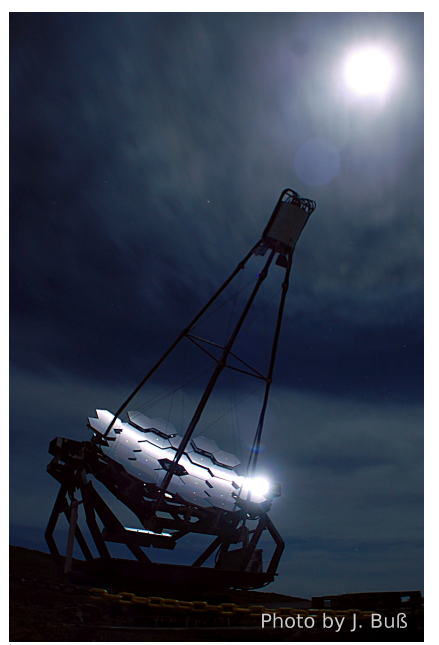

Figure 1. FACT-Observation during strong moon light.

Since summer 2012, FACT has been operated remotely without the need for a data-taking crew onsite. Automation has constantly improved, and FACT is currently switching from remote to robotic operation. For information about the design of FACT, see [1].

One of the major goals of FACT was the proof of principle for the use of SiPM in Cherenkov astronomy. Over the last five years, the operation of FACT has proven that SiPM show a stable performance over a large temperature range and under various light conditions. The operation is possible even during full moon without any filter, which is not possible with photomultiplier tubes, which are currently used in IACT. This allows an increase in the duty cycle of the telescope to a maximum, only limited by weather conditions. The performance of the sensors is described in detail in Section 2 and in [2], and the performance in respect to physics questions is described in Section 3 and in [3].

The major physics goal of the FACT project is the long-term monitoring of bright TeV blazars in the northern hemisphere. Hence, an unbiased and densely sampled data set of blazars is obtained, independent of the current activity level of the sources. The concept of the long-term monitoring is illustrated in more detail in Section 4.

\section{Performance of the Photo Sensors of FACT}

In the last five years, the FACT collaboration investigated the performance of the novel photo sensors. A single photon equivalent spectrum (shown in Figure 2) was obtained from dark counts. The shown spectrum is a sum spectrum of all 1440 pixels of the camera. A resolution of one photon is clearly visible, and entries up to 7 photon equivalent (p.e.) are visible in the spectrum. 
The gain of the individual pixels is extremely homogeneous over the whole camera. The standard deviation over the whole experienced range of outside temperature conditions and over the full camera is less than $2.3 \%$, and not a single sensor has shown an ageing effect so far.

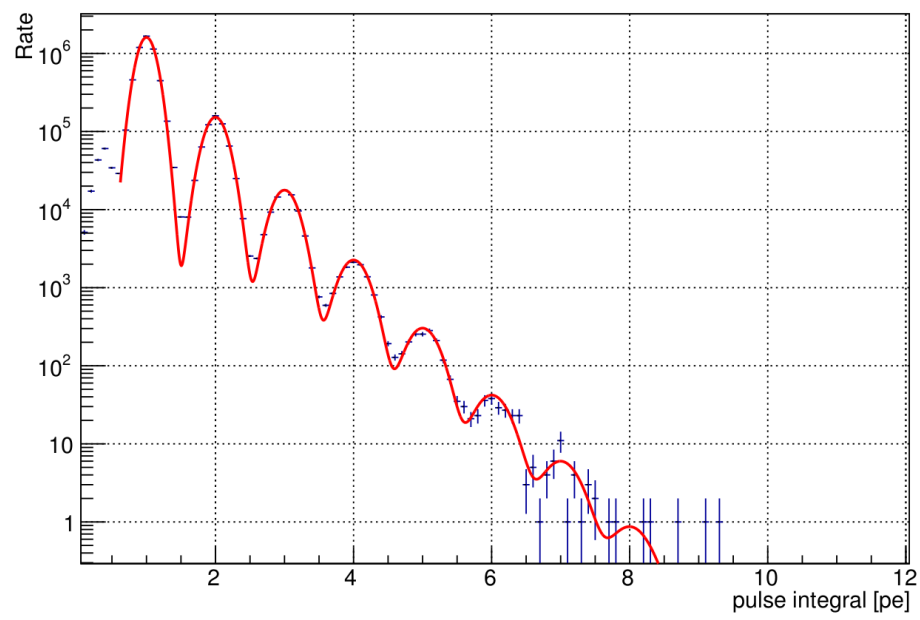

Figure 2. Single photon equivalent spectrum (blue), obtained from dark counts. Overlayed is a fit of a modified Erlang distribution (solid red line). The spectrum includes all 1440 pixels of the camera. More details in [2].

Figure 3 shows different scans (so-called ratescans) of the triggerrate of the telescope versus the applied threshold of the trigger. The ratescans show two different branches: one dominated by triggers caused by night sky background light, one dominated by triggers caused by cosmic ray showers. The similarity between ratescans from 2012 (black) and 2015 (green) under the same light conditions indicates an excellent longterm performance of the trigger behavior and of the sensors of the telescope. In addition, Figure 3 shows that the cosmic ray trigger rate branch in the ratescans is independent of light conditions. It is even possible to directly track the full moon and trigger air showers (see [4] for details).

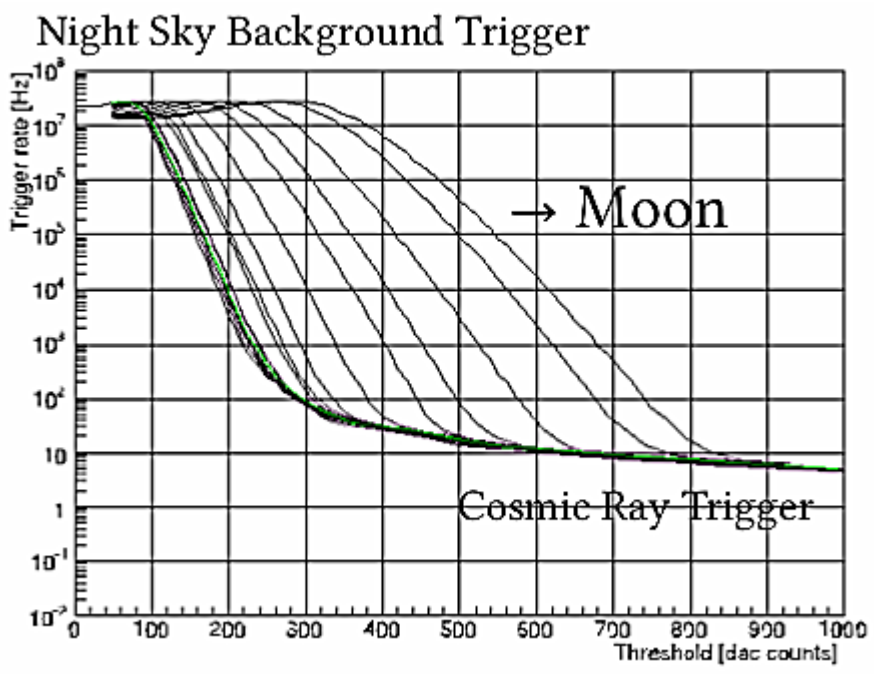

Figure 3. Ratescans (scans of the trigger rate versus the applied trigger threshold) under different light conditions. Black: 2012, Green: 2015. 


\section{Physics Performance of FACT}

To evaluate the performance of FACT with respect to energy bias, energy resolution, and energy range, an analysis of data of the Crab Nebula was performed [3]. Overall, $87.6 \mathrm{~h}$ of data with zenith angles below $30^{\circ}$ and under dark night and good observation conditions were analysed. The applied data analysis chain based on modern data mining methods was developed in cooperation with computer scientists within the scope of the Collaborative Research Center 876 at the TU Dortmund. The preprocessing of the raw data was performed with FACT-Tools [5], an extension of the streams-framework [6].

A random forest regression [7] is used to estimate the energy of the primary particle. The evaluation of the energy bias and energy resolution is based on this estimated energy.

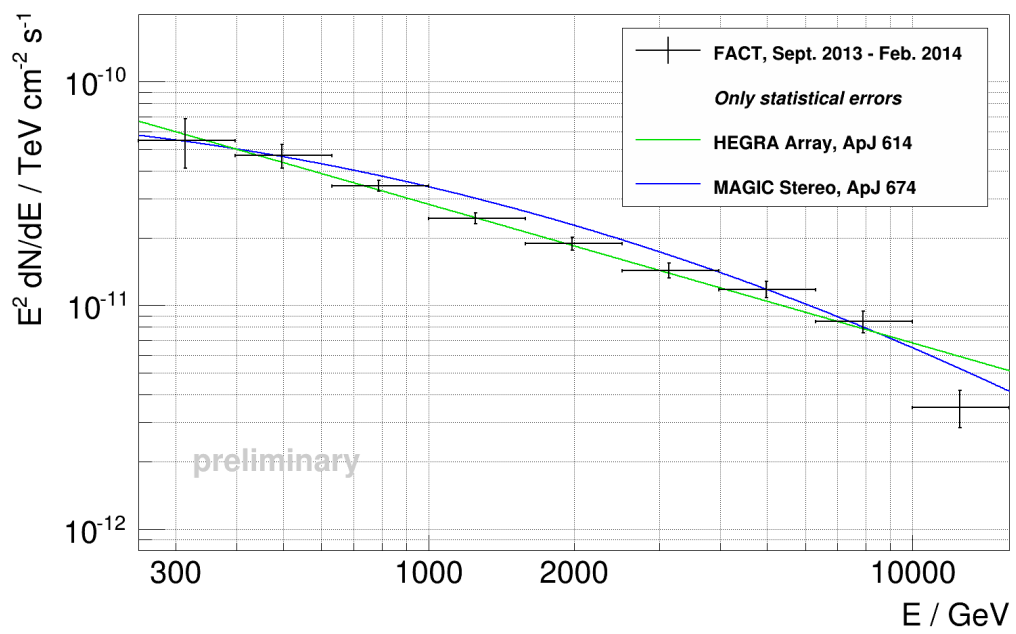

Figure 4. Unfolded energy spectrum, scaled with the square of the energy $E$ of the Crab Nebula [3], compared to HEGRA [8] and MAGIC [9].

For most of the dynamic range of the telescope, the energy resolution is about $22 \%$; see [3] for details. For the background suppression, a random forest classification [10] was used. The resulting gamma data set was unfolded using a Tikhonov Regularized Unfolding with the unfolding software TRUEE [11], to obtain the energy spectrum of the Crab Nebula. The Crab Nebula could be detected with a significance of $43.54 \sigma$. The energy spectrum-scaled with the square of the energy $E$-is shown in Figure 4. Another independent analysis chain based on the Mars CheObs framework [12] yielded similar results.

\section{Monitoring Blazars at TeV Energies}

FACT is monitoring a set of bright blazars in the Northern sky. A Quick Look Analysis (QLA) based on the Mars CheObs framework [12] processes the taken data immediately after acquisition. The preliminary results (uncorrected for the effect of different zenith angle and changing light condition) are publicly available with short latency: http://www.fact-project.org/monitoring/.

The QLA has been running since December 2012. The results up to July 2016 are displayed in Figure 5. The data points show daily binned excess rates for the four most observed sources measured by FACT.

During their visibility windows, the sources are observed independently of their activity state. Even if the source does not show a significant signal on a daily timescale, the monitoring continues. 


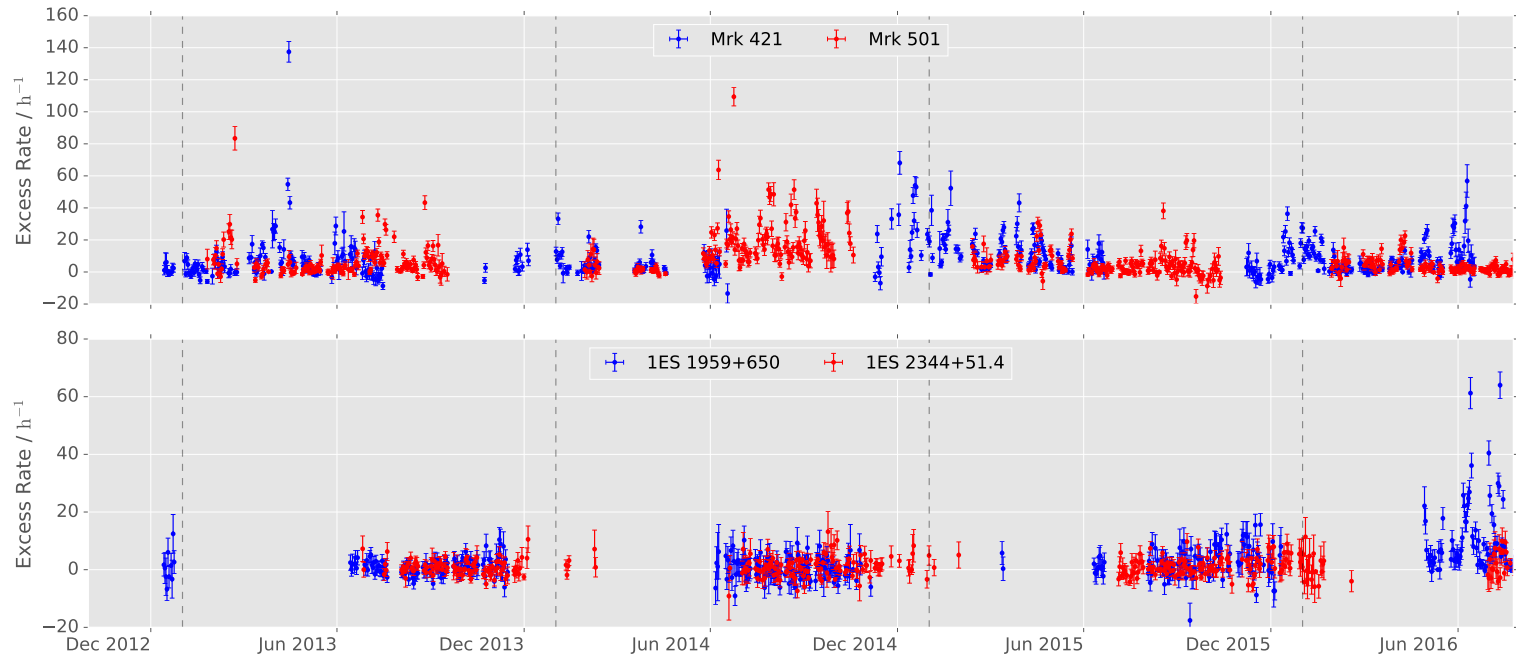

Figure 5. Daily binned excess rate curves for the main sources measured by FACT. The sources are monitored during the visibility periods each year. Holes during these visibilty periods are mainly caused by bad weather conditions.

\section{Flare Alerts}

To ensure multi-wavelength observations, other instruments are alerted in case of enhanced activity of one of the monitored sources. Hence, the excess rates of the observed sources-calculated by the QLA - are monitored, and an alert is raised if one of the rates exceeds predefined limits. Between the start of the official flare alert system in March 2014 and July 2016, FACT sent 31 flare alerts. Figure 6 visualizes the sent flare alerts (vertical lines). To improve the clarity, the data is binned daily, whereas the condition for raising a flare alert is based on a minimum of $20 \mathrm{~min}$ of data. The prefined limit for Markarian 421 and Makarian 501 is 60 events/h, which corresponds to a flux level of roughly $3 \mathrm{Crab}$ Units. The limit for other sources is roughly 0.5 Crab Units.

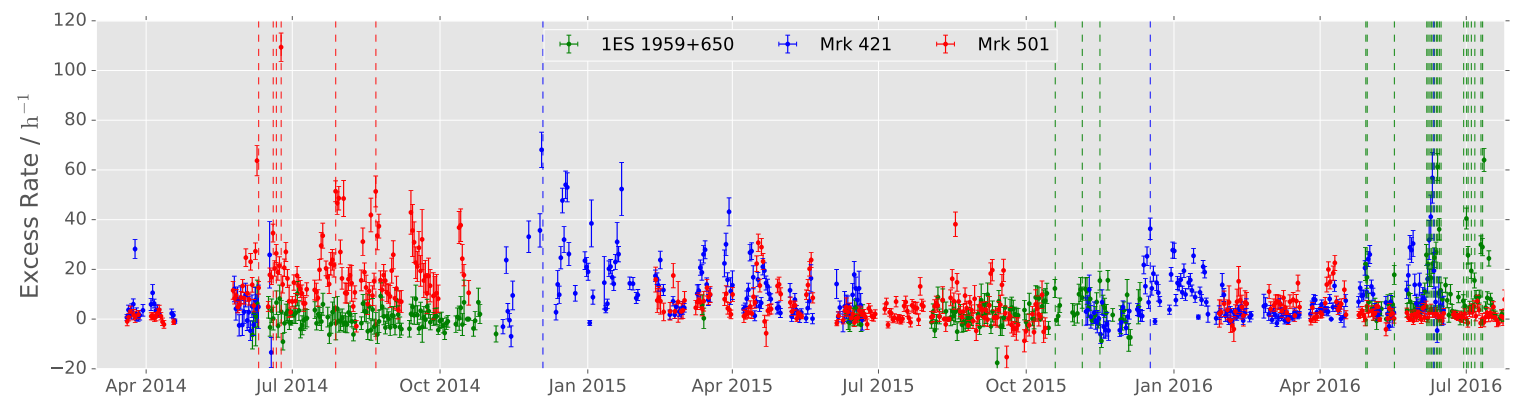

Figure 6. Excess rate curve for the three sources which FACT has sent flare alerts for. The flare alerts are marked with vertical lines.

After a flare alert of FACT for Markarian 501 in June 2014, the H.E.S.S. telescopes performed follow-up observations (see ATel \#6268). In December 2015, a flare alert from FACT for Markarian 421 triggered Target-of-Opportunity observations with INTEGRAL and Swift. For the source Markarian 421, a joint ATel \#9137 with HAWC and Swift was sent in June 2016. For the source 1ES 1959+650, four ATel were sent (\#9010, \#9139, \#9148, and \#9203) in April, June, and July 2016. Three of them were joint ATel with MAGIC (\#9203) and with Fermi-LAT, MAGIC, and VERITAS (\#9010 and \#9148). For ATel \#9148, Swift was also involved. 


\section{Lightcurve of Markarian 501 in Summer 2014}

As an example of the advantages of longterm monitoring of bright blazars, Figure 7 shows the light curve of Markarian 501 in Summer 2014. To improve the clarity, the data is binned in $60 \mathrm{~min}$ bins. During the shown time window, five flaring activities could be caught in total. The highest flare on 24.06.2014 is shown in more detail and with 20 min binning in the upper part of Figure 7. In addition to the excess rates during the flare, the unbiased monitoring also adds information about the activity level before and after the flaring activities. As the observation is independent of the activity level and longer than in the cases of large IACT (up to $6 \mathrm{~h}$ ), the opportunity to catch rising and falling edges of the flares is increased. The densely sampled light curve (only restricted by poor weather conditions) also provides a large overlap for possible multi-wavelength observations for both high and low state activities on minutes to hours timescale. For example, for a correlation study between very high energy VHE gamma ray fluxes and other wavelengths, long-term monitoring provides robust statistics for the detection of possible correlations.

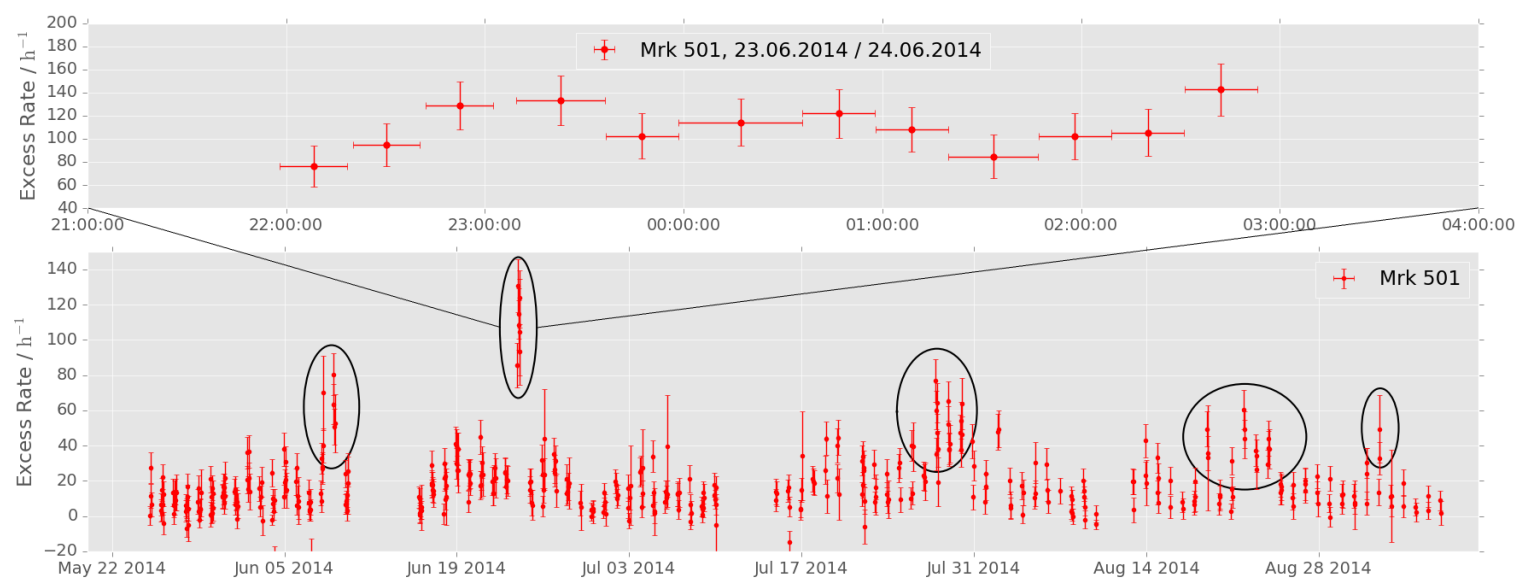

Figure 7. Excess rate curve for Markarian 501 in summer 2014. The highest flare is shown in more detail. Flaring states are marked with black ellipses. The bins correspond to observation windows with an effective ontime of $20 \mathrm{~min}$ (top) and $60 \mathrm{~min}$ (bottom). The effective ontime is the observation time with enabled trigger.

\section{Conclusions}

The five years of successful operation of FACT successfully proves the suitability of SiPM for Cherenkov Gamma Ray astronomy. The homogeneous and stable performance of the photo sensors over the whole time range and for various light conditions allows for a densely sampled and unbiased long-term monitoring of bright $\mathrm{TeV}$ blazars in the Northern sky. The evaluation of the physics performance of the telescope and the analysis chain enables the production of an energy spectrum of the Crab Nebula ranging from $250 \mathrm{GeV}$ up to $16 \mathrm{TeV}$. An energy resolution of roughly $22 \%$ for energies above $600 \mathrm{GeV}$ is achieved, with an improving resolution for energies above $10 \mathrm{TeV}$.

From first light in October 2011 until July 2016, FACT acquired roughly $1400 \mathrm{~h}$ ontime for Markarian 421, $1650 \mathrm{~h}$ for Markarian 501, $650 \mathrm{~h}$ for 1ES 1959+560, $830 \mathrm{~h}$ for 1ES 2344+51.4, and 1200 $\mathrm{h}$ for the Crab Nebula. The monitoring of the sources enabled FACT to sent 31 flare alerts between March 2014 and July 2016 to the astroparticle community, resulting in several follow-up observations of other experiments. The unbiased light curves allow for variability studies of the sources and correlation studies with other multi-wavelength observations, contributing to the revealing of the physical properties of blazars and the particle acceleration in these violent sources. 
Acknowledgments: The important contributions from ETH Zurich grants ETH-10.08-2 and ETH-27.12-1 as well as the funding by the German BMBF (Verbundforschung Astro- und Astroteilchenphysik) and HAP (Helmoltz Alliance for Astroparticle Physics) are gratefully acknowledged. We are thankful for the very valuable contributions from E. Lorenz, D. Renker and G. Viertel during the early phase of the project. We thank the Instituto de Astrofisica de Canarias allowing us to operate the telescope at the Observatorio del Roque de los Muchachos in La Palma, the Max-Planck-Institut für Physik for providing us with the mount of the former HEGRA CT 3 telescope, and the MAGIC collaboration for their support. Part of this work is supported by Deutsche Forschungsgemeinschaft (DFG) within the Collaborative Research Center SFB 876 "Providing Information by Research-Constrained Analysis", project C3, and within the Collaborative Research Center SFB 823 "Statistical modelling of nonlinear dynamic processes", project C4.

Conflicts of Interest: The authors declare no conflict of interest.

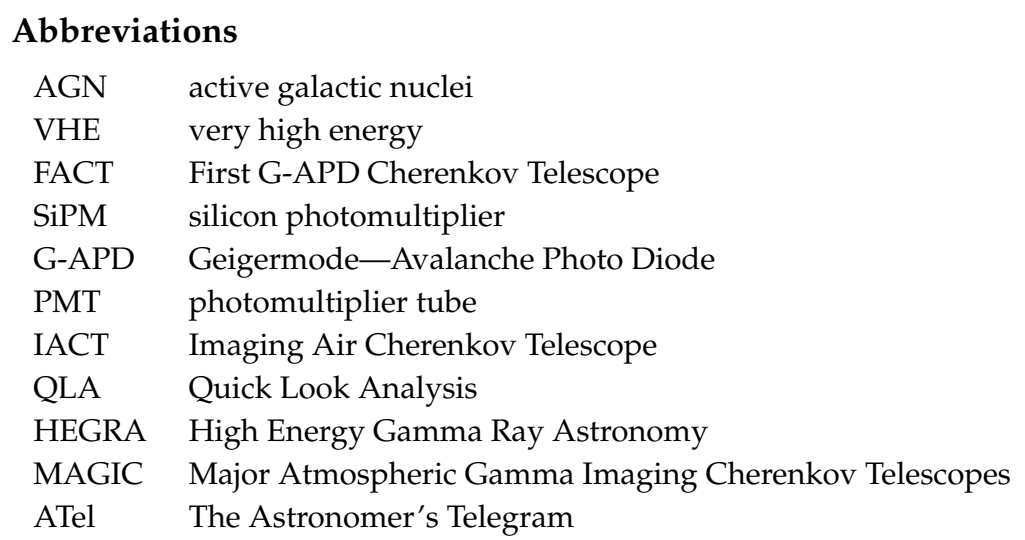

\section{References}

1. Anderhub, H.; Backes, M.; Biland, A.; Boccone, V.; Braun, I.; Bretz, T.; Buß, J.; Cadoux, F.; Commichau, V.; Djambazov, L.; et al. Design and operation of FACT-The first G-APD Cherenkov telescope. J. Instrum. 2013, 8, P06008.

2. Biland, A.; Bretz, T.; Buß, J.; Commichau, V.; Djambazov, L.; Dorner, D.; Einecke, S.; Eisenacher, D.; Freiwald, J.; Grimm, O.; et al. Calibration and performance of the photon sensor response of FACT- the first G-APD Cherenkov telescope. J. Instrum. 2014, 9, P10012.

3. Temme, F. FACT-First Energy Spectrum from a SiPM Cherenkov Telescope. In Proceedings of the 34th International Cosmic Ray Conference, Hague, The Netherlands, 30 July-6 August 2015.

4. Knötig, M.L.; Biland, A.; Bretz, T.; Buß, J.; Dorner, D.; Einecke, S.; Eisenacher, D.; Hildebrand, T.; Krähenbühl, T.; Lustermann, W.; et al. FACT-Long-term stability and observations during strong Moon light. In Proceedings of the 33rd International Cosmic Ray Conference, Rio de Janeiro, Brazil, 2 July-9 July 2013.

5. Brügge, K. FACT-Tools: Streamed Real-Time Data Analysis. In Proceedings of the 34th International Cosmic Ray Conference, Hague, The Netherlands, 30 July-6 August 2015.

6. Bockermann, C. Mining Big Data Streams for Multiple Concepts. Ph.D. Thesis, TU Dortmund, Dortmund, Germany, 2015.

7. Pedregosa, F.; Varoquaux, G.; Gramfort, A.; Michel, V.; Thirion, B.; Grisel, O.; Blondel, M.; Prettenhofer, P.; Weiss, R.; Dubourg, V.; et al. Scikit-learn: Machine Learning in Python. J. Mach. Learn. Res. 2011, 12, 2825-2830.

8. Aharonian, F.; Akhperjanian, A.; Beilicke, M.; Bernlöhr, K.; Börst, H.G.; Bojahr, H.; Bolz, O.; Coarasa, T.; Contreras, J.L.; Cortina, J.; et al. The Crab Nebula and Pulsar between $500 \mathrm{GeV}$ and $80 \mathrm{TeV}$ : Observations with the HEGRA Stereoscopic Air Cerenkov Telescopes. Astrophys. J. 2004, 614, 897.

9. Aleksić, J.; Ansoldi, S.; Antonelli, L.A.; Antoranz, P.; Babic, A.; Bangale, P.; Barceló, M.; Barrio, J.L.; Becerra González, J.; Bednarek, W.; et al. The major upgrade of the $\{$ MAGIC $\}$ telescopes, Part II: A performance study using observations of the Crab Nebula. Astropart. Phys. 2016, 72, 76-94.

10. Mierswa, I.; Wurst, M.; Klinkenberg, R.; Scholz, M.; Euler, T. YALE: Rapid Prototyping for Complex Data Mining Tasks. In Proceedings of the 12th ACM SIGKDD, Philadelphia, PA, USA, 20-23 August 2006. 
11. Milke, N.; Doert, M.; Klepser, S.; Mazin, D.; Blobel, V.; Rhode, W. Solving inverse problems with the unfolding program TRUEE: Examples in astroparticle physics. Nucl. Instr. Meth. Phys. Res. A 2013, 697, 133-147.

12. Bretz, T.; Dorner, D. MARS-CheObs ed.: A flexible software framework for future Cherenkov telescopes. In Proceedings of the 11th ICATPP Conference, Como, Italy, 5-9 October 2009.

(C) 2017 by the authors; licensee MDPI, Basel, Switzerland. This article is an open access article distributed under the terms and conditions of the Creative Commons Attribution (CC BY) license (http:/ / creativecommons.org/licenses/by/4.0/). 